\title{
LA ENSEÑANZA DE LA ESCRITURA CIENTÍFICO-ACADÉMICA EN INGLÉS EN EL POSGRADO: REFLEXIONES SOBRE LA PRÁCTICA
} ACADEMIC WRITING INSTRUCTION IN ENGLISH IN
GRADUATE SCHOOL: REFLECTIONS ON PRACTICE Viviana Innocentini*

Rec.: 13-09-2021. Acept.: 12-10-2021. Publ.: 20-12-2021

DOI: https://doi.org/10.29035/ucmaule.61.121

\section{RESUMEN}

La escritura es una habilidad fundamental en la universidad, tanto a nivel de grado como de posgrado. En el posgrado, específicamente, el desarrollo de la escritura científico-académica no se restringe únicamente a la primera lengua; dado el rol del inglés como lingua franca de la comunicación científica, los estudiantes de posgrado deben ser capaces de producir géneros expertos también en esa lengua. En Argentina, muchas instituciones universitarias demandan producciones escritas en inglés como requisitos previos a la graduación en carreras de maestría y doctorado, pero son pocas las instituciones que capacitan a sus estudiantes en la escritura de géneros expertos en inglés. En este trabajo me propongo analizar la aplicabilidad de un enfoque críticopragmático basado en géneros discursivos para la enseñanza de la escritura de géneros expertos en inglés en el nivel de posgrado en una universidad argentina. A la luz de la literatura existente y partiendo de un análisis empírico, situado y de corte cualitativo, este trabajo da cuenta de tres iniciativas docentes tendientes a mediar la brecha existente entre las demandas de escritura en inglés y la enseñanza de escritura que favorezca la participación genuina y efectiva de escritores multilingües en una comunidad internacional.

* Universidad Nacional de Mar del Plata https://orcid.org/0000-0001-7102-8340 vinnocentini@mdp.edu.ar 
Innocentini, V. (2021). La enseñanza de la escritura científico-académica en inglés en el posgrado: Reflexiones sobre la práctica. UCMaule, 67, julio-diciembre, 121-133. DOI: https://doi.org/10.29035/ucmaule.61.121

Palabras Clave: escritura científico-académica, inglés, géneros discursivos, práctica docente.

\section{ABSTRACT}

Writing is an essential skill in higher education, both at undergraduate and graduate levels. In graduate school, academic writing is not only restricted to the first language; given the role of English as the lingua franca of scientific communication, graduate students must be able to produce expert genres in English as well. In Argentina, written productions in English are often prerequisites for graduation in master's and doctoral studies, but few institutions offer training on the writing of expert genres in the foreign language. In this paper, I reflect upon the applicability of a critical-pragmatic genre approach for the teaching of expert genres in English at the postgraduate level in an Argentinian university. In the light of the existing literature and based upon empirical, situated and qualitative analysis, this work reports on three teaching initiatives aimed at mediating the gap between the demands of writing in English and academic writing instruction that favors genuine and effective participation of multilingual writers in an international community.

Key words: academic writing, English, genres, teaching practice

\section{INTRODUCCIÓN}

La escritura es sin dudas una habilidad inherente al aprendizaje universitario (Creme \& Lea, 2008; Gómez Espinoza et al., 2020); a través de ella se establece gran parte de la comunicación entre profesores y estudiantes, se apropian saberes y se da cuenta del conocimiento disciplinar adquirido. En el nivel de posgrado, la escritura no sólo es el medio a través del cual los estudiantes, investigadores nóveles, a menudo son evaluados, sino que es además el medio para la obtención de recursos para realizar investigaciones y difundirlas, tanto dentro como fuera de las fronteras institucionales. La escritura científico-académica es, por sobre todo ello, una herramienta fundamental para que los estudiantes puedan adentrarse en la cultura disciplinar (Lillis, 2001) y en las formas particulares de construcción y difusión de conocimiento en sus disciplinas. Da cuenta de tal importancia, el desarrollo de movimientos como la Escritura a través del currículo, Escritura en las disciplinas y Alfabetización o Literacidades Académicas (Bazerman et al., 2005; Bazerman et al., 2016; Carlino, 2013; Molina Natera, 2012; Russell, 
Innocentini, V. (2021). La enseñanza de la escritura científico-académica en inglés en el posgrado: Reflexiones sobre la práctica. UCMaule, 67, julio-diciembre, 121-133. DOI: https://doi.org/10.29035/ucmaule.61.121

2002) y el auge de investigaciones e iniciativas pedagógicas centradas en las prácticas letradas académicas o científico-académicas con un gran impacto para la formación universitaria de grado y posgrado en distintos países de América Latina (Colombo \& Carlino, 2015; Navarro, 2019; Parodi, 2010).

En el nivel de posgrado, específicamente, el desarrollo de la escritura científicoacadémica no se restringe únicamente a una primera lengua o lengua dominante. Dado que el inglés se ha transformado en la lingua franca de la comunicación científica, los estudiantes de carreras de posgrado deben ser capaces de escribir también en esta lengua. En muchos casos, la escritura científico-académica en inglés en revistas de amplia trayectoria no solamente responde al deseo de investigadores nóveles de visibilizar sus investigaciones en una comunidad internacional, sino que representa incluso un requisito previo a la obtención de títulos de nivel de maestría y doctorado (Carrasco et al., 2011; Corcoran \& Englander, 2016). En muchas universidades argentinas, sin embargo, los cursos académicos curriculares de posgrado abordan mayormente las habilidades de comprensión lectora, pero no tienen en cuenta la escritura en inglés, o bien, a menudo son de carácter propedéutico y enmascaran un foco en aspectos lingüístico-gramaticales que no permiten dar cuenta de la escritura como práctica social, discursiva, situada (Cassany \& Morales, 2009). Aunque son pocos los antecedentes existentes, en los últimos años se han desarrollado y afianzado algunas iniciativas pedagógicas potentes respecto de la enseñanza de la escritura en inglés con fines de publicación en el nivel universitario (Waigandt et al., 2019), tendientes a favorecer el proceso de inserción de investigadores argentinos en una comunidad científica internacional, respondiendo a las expectativas de la comunidad discursiva, pero desarrollando, a su vez, sus propias voces como escritores (Castelló \& Donhaue, 2012).

Un concepto central para abordar el estudio y la enseñanza de la escritura científicoacadémica en inglés es el de género discursivo, en tanto permite vincular los textos producidos por los escritores con los contextos específicos de producción y circulación, incluyendo la relación entre las convenciones y expectativas existentes y las decisiones y objetivos retórico-pragmáticos de los escritores. Según Bajtín (2005), los géneros discursivos son enunciados relativamente estables que pertenecen o se insertan en la praxis $y$, por tanto, dan cuenta de ciertas condiciones específicas del contexto que los posibilitan y determinan; existe a su vez una dimensión individual y una social, colectiva, que da cuenta de las regularidades o convenciones existentes en términos de estilo, objetivos y recursos que permiten que los participantes de esas distintas esferas los reconozcan y validen. En otras palabras, la producción de ciertas 
Innocentini, V. (2021). La enseñanza de la escritura científico-académica en inglés en el posgrado: Reflexiones sobre la práctica. UCMaule, 67, julio-diciembre, 121-133. DOI: https://doi.org/10.29035/ucmaule.61.121

clases de textos, en tanto representativos de cierto género discursivo presupone una "tensión entre la repetición y la creatividad" (Navarro, 2019, p. 8), o entre las elecciones lingüístico-discursivas propias de cada escritor y los rasgos compartidos y esperados por la comunidad discursiva en la que circulan.

Una serie de ventajas han sido identificadas en torno a un enfoque basado en los géneros discursivos para la enseñanza de la escritura académica, extrapolables a la enseñanza de la escritura de géneros expertos en el nivel de posgrado en una lengua extranjera. Hyland (2004) sostiene que además de identificar las necesidades concretas y reales de los estudiantes, un enfoque basado en los géneros discursivos, en tanto explícito y sistemático, permite visibilizar los objetivos retóricos y los patrones discursivos existentes y contribuye, así, a la generación de conciencia por parte de docentes y estudiantes tanto de las convenciones como de las posibilidades de realización lingüística existentes; abordados de manera no prescriptiva sino analíticoreflexiva, los géneros permiten a su vez identificar posibilidades de variación y representan, en tal sentido, una herramienta para desafiar los discursos dominantes. De forma similar, Navarro (2019) argumenta que la enseñanza de la escritura basada en los géneros discursivos representa una aproximación "significativa, articulada, socioconstructivista, crítica y explícita" (p. 23) a los géneros no expertos, o en formación. Pese a posibles distancias geopolíticas o culturales, ambos investigadores dan cuenta de la potencia de este enfoque.

En lo que respecta específicamente a la enseñanza de la escritura de géneros expertos en inglés a hablantes multilingües, el análisis crítico de los géneros discursivos (MottaRoth, 2008) ha sido identificado como una herramienta para disminuir la brecha existente entre las expectativas de los llamados gatekeepers, editores, revisores, científicos en las esferas centrales de la academia, y las realidades que enfrentan muchos de quienes intentan participar desde la periferia, con escasos recursos económicos, ausencia de un proceso de enculturación disciplinar, desconocimiento de la lengua extranjera y de los aspectos genérico-discursivos que atraviesan la escritura. En este contexto, Corcoran y Englander $(2016,2019)$ proponen abordar la escritura desde una perspectiva crítico-pragmática (Corcoran, 2019; Curry \& Lillis, 2013) que recupere aspectos importantes de la comunicación científica en inglés no siempre abordados, como las asimetrías sociopolíticas y la diversidad lingüística. En otras palabras, un enfoque crítico-pragmático supone un trabajo empírico, contextualizado y sistematizado con los géneros y comunidades discursivas, que permita identificar movidas retóricas y elecciones retórico-lingüísticas, pero, a su vez, reflexionar abierta 
Innocentini, V. (2021). La enseñanza de la escritura científico-académica en inglés en el posgrado: Reflexiones sobre la práctica. UCMaule, 67, julio-diciembre, 121-133. DOI: https://doi.org/10.29035/ucmaule.61.121

y explícitamente respecto de las implicancias de determinadas elecciones, de las relaciones hegemónicas construidas en el discurso y de las posibilidades concretas de los investigadores multilingües de desarrollar sus propias voces como escritores, ya sea amalgamando sus prácticas de escritura a los patrones dominantes o desafiándolos.

A la luz de los antecedentes mencionados (Waigandt et al., 2019) y en línea con las propuestas de Corcoran y Englander (2016) y Navarro (2019), pretendo en este trabajo reflexionar respecto de la aplicabilidad de un enfoque crítico-pragmático basado en géneros discursivos para la enseñanza de la escritura de géneros expertos en inglés, en el nivel de posgrado en una universidad argentina. En primer lugar, proporciono una descripción general del contexto específico y de las necesidades de intervención identificadas. Asimismo, pretendo analizar fortalezas y debilidades identificadas a lo largo de un proceso de transición entre tres propuestas de intervención pedagógica realizadas a lo largo de los años y a la luz de las experiencias y representaciones docentes sobre la escritura en inglés, pero, a su vez, retroalimentadas por los aportes de estudiantes de posgrado. Espero que este análisis situado, empírico y de corte cualitativo sea un aporte para contribuir a mediar la brecha existente entre las demandas de escritura en inglés y la enseñanza de escritura que favorezca la participación genuina y efectiva de escritores multilingües en una comunidad internacional.

\section{Descripción del contexto: importancia de la investigación y la escritura de géneros científico-académicos expertos}

Desde su creación, la Facultad de Ciencias Agrarias de la Universidad Nacional de Mar del Plata (UNMdP) ha estado estrechamente vinculada a la investigación; por un lado, en términos edilicios, funciona junto a una estación experimental del Instituto Nacional de Tecnología Agropecuaria (INTA), con la que conforma hace más de seis décadas la denominada "Unidad Integrada". Esta supone no solo el uso de recursos compartidos sino el trabajo mancomunado de profesionales cuyas funciones migran entre ambas instituciones desde aquel entonces y determinan, en gran medida, una fuerte impronta de la investigación en la currícula. Se ofrecen en la actualidad varias carreras de posgrado en los niveles de especialización, maestría y doctorado; estas últimas, a menudo recurren al análisis de textos científico-académicos en inglés para la enseñanza y demandan producciones escritas en la lengua extranjera, en forma de abstract y/o artículo de investigación como requisitos previos a la graduación.

Para apoyar a los estudiantes en los procesos de elaboración y escritura de sus proyectos de investigación, a lo largo de los años se ha ofrecido un curso de carácter obligatorio 
Innocentini, V. (2021). La enseñanza de la escritura científico-académica en inglés en el posgrado: Reflexiones sobre la práctica. UCMaule, 67, julio-diciembre, 121-133. DOI: https://doi.org/10.29035/ucmaule.61.121

denominado "Módulo humanístico: Encuadre metodológico para la redacción científica". Sin embargo, pese a los requerimientos de producciones en inglés, no existen a la fecha asignaturas curriculares para formar a estudiantes de posgrado en la escritura de los géneros expertos que les son demandados, a excepción de una propuesta surgida a partir de una serie de iniciativas docentes (Figura 1) que pretendo discutir aquí.

Figura 1: Iniciativas docentes para la enseñanza de la escritura de géneros expertos en inglés a estudiantes de posgrado en la FCA, UNMDP.

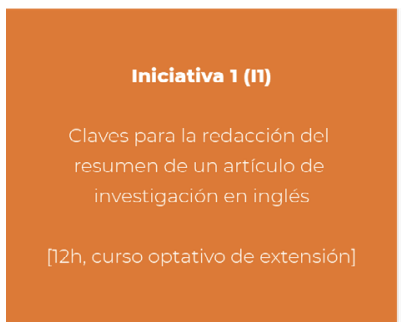

\section{Iniciativa 2 (12)}

Introducción a la escritura científica en inglés

[32h, curso extracurricular de posgrado]
Iniciativa 3 (13)

Escritura cientifico académica en inglés

[32h, curso particular de posgrado]

Como se ilustra en la figura 1, las tres iniciativas corresponden a propuestas docentes para la enseñanza de la escritura de géneros expertos en inglés originadas en respuesta a las necesidades identificadas e implementadas de forma secuencial desde 17 a 13. Cada nueva iniciativa se cimentó en la propuesta anterior y, a lo largo de la transición, se buscó profundizar el abordaje de la escritura en tanto práctica social, situada y contextualizada. Si bien durante el desarrollo de cada iniciativa se realizaron cambios menores, en función de una evaluación en proceso, los ajustes o adecuaciones en los programas de cada propuesta fueron realizados en forma posterior, dado que involucraron, además, procesos de intercambio con autoridades de la facultad y la universidad y, en el caso de 13, con instituciones de evaluación y acreditación externas. Las modificaciones más sustanciales en cada propuesta docente se realizaron sobre la base del curso anterior y se justificaron en procesos de evaluación y retroalimentación tanto formativas como sumativas, que incluyeron la autoevaluación docente, la evaluación de los aprendizajes y las perspectivas de los estudiantes respecto del curso en cuestión y de la escritura en inglés, expresadas informalmente y recolectadas también a través de encuestas previas y posteriores a cada propuesta.

El curso "Claves para la redacción del resumen de un artículo científico en inglés" (17) representó la primera iniciativa tendiente a mediar en la brecha existente entre las demandas de escritura en la lengua extranjera en el posgrado y la oferta académica institucional. Surgió como un breve curso optativo de extensión universitaria de 12 
Innocentini, V. (2021). La enseñanza de la escritura científico-académica en inglés en el posgrado: Reflexiones sobre la práctica. UCMaule, 67, julio-diciembre, 121-133. DOI: https://doi.org/10.29035/ucmaule.61.121

horas reloj enmarcado en el eje "comunicando ciencia" del programa Universidad de Verano de la UNMdP. Después de algunos años, esa propuesta inicial fue reformulada y devino en un curso extracurricular de posgrado denominado "Introducción a la escritura científica en inglés" (12) con una duración de 32 horas reloj. Aunque ambas iniciativas toman al género abstract como el pilar sobre el cual se desarrolla la escritura en inglés, existen, entre estas propuestas, diferencias sustanciales, que permiten dar cuenta de algunas reflexiones teórico-metodológicas importantes a la luz de la literatura existente.

Posteriormente, y con un fuerte anclaje en las experiencias del curso anterior, se llevó adelante una iniciativa para curricularizar la enseñanza de la escritura en inglés, con el apoyo de la Comisión de posgrado de la facultad. En el marco de un proceso de adecuación de un programa de maestría y de acreditación de esta ante la Comisión Nacional de Evaluación y Acreditación Universitaria (CONEAU), logró plasmarse una nueva propuesta didáctica titulada "Escritura científico-académica en inglés" (13), que retoma y complejiza los alcances del curso que le diera origen, a la vez que propone un trabajo articulado con otros géneros de la misma red o grupo (Navarro, 2019). Una serie de factores confluyeron e influyeron en este trabajo; la estrecha relación entre la docencia en el nivel de posgrado y los avances en mi investigación doctoral, sumado a un proceso propio de enculturación y socialización' con colegas de disciplinas afines sin dudas funcionaron como andamiaje y contribuyeron en la transición hacia la iniciativa más reciente (I3).

\section{Reflexiones en torno a las iniciativas docentes}

Retomando el aporte realizado por Navarro, intentaré dar cuenta del proceso de transición hacia una propuesta didáctica para la escritura en inglés basada en géneros discursivos que contempla las características centrales identificadas por el autor: es "significativa, articulada, socio-constructivista, crítica y explícita" (Navarro, 2019, p. 23).

Una didáctica basada en géneros discursivos es, en esencia, significativa en tanto responde a las necesidades e intereses genuinos de los estudiantes, vinculados a sus contextos académicos y profesionales, y "hace que los aprendizajes y enseñanzas tengan sentido para los estudiantes" (Navarro, 2019, p. 23). En este aspecto, la transición

\footnotetext{
$1 \quad$ El proceso de enculturación y socialización al que refiero se vincula estrechamente con mis estudios doctorales en Lingüística en la Universidad de Buenos Aires. En forma paralela y complementaria al proceso de indagación propio de mi investigación sobre escritura académica, específicamente sobre el uso del metadiscurso en el género abstract en inglés y en español, por iniciativa de mi director, Dr. Federico Navarro, se creó un grupo de tesistas de disciplinas afines (Educación, Lingüística, Comunicación social, Psicología) y se comenzaron a desarrollar bimensualmente reuniones para compartir experiencias, discutir aspectos teóricos y metodológicos, socializar avances, en muchos casos además con la presencia de investigadores externos. Cada uno de estos intercambios, indudablemente aportó para lograr una mirada menos atomizada de un fenómeno complejo como es la escritura.
} 
Innocentini, V. (2021). La enseñanza de la escritura científico-académica en inglés en el posgrado: Reflexiones sobre la práctica. UCMaule, 67, julio-diciembre, 121-133. DOI: https://doi.org/10.29035/ucmaule.61.121

desde 17 a 13 ha posibilitado ajustar la propuesta didáctica inicial, centrada en el análisis de abstracts escritos en inglés como herramienta para facilitar la escritura en dicha lengua, y ampliarla para incluir además su relación con otros géneros discursivos de la misma red o grupo. Así, por ejemplo, aspectos no abordados en la propuesta inicial (17) vinculados a los contextos de circulación y funciones comunicativas específicas de los abstracts que acompañan un artículo de investigación en contraste con los enviados a un evento científico se incorporan como contenidos obligatorios del curso extracurricular (12). Sumado a ello, esta segunda propuesta plantea un trabajo de análisis exploratorio de las normas de publicación o estilo de cada contexto de escritura antes mencionado. Se analizan y discuten en clase algunos ejemplos de normas o guías para autores, preseleccionadas a priori con fines pedagógicos, aspecto sobre el cual se trabajó en 13 con el objeto de anclar aún más los aprendizajes a los contextos posibles de escritura en inglés de cada estudiante. En este sentido, en 13 son los propios estudiantes de posgrado quienes seleccionan destinos posibles para sus producciones en inglés (revistas y eventos científicos, bases de datos institucionales o eventos académicos) y ajustan tales producciones en la lengua extranjera a los requerimientos explicitados en los destinos identificados. Se ofrecen en los encuentros espacios de diálogo para socializar y discutir normas, identificar aspectos centrales comunes y/o específicos de cada contexto y se (re)editan manuscritos a partir de procesos de autoevaluación y evaluación de pares. Finalmente, en relación a los géneros propiamente dichos, esta última propuesta no sólo incluye la escritura de abstracts sino que incorpora además al género artículo de investigación. Se recurre a un análisis minucioso de los propósitos retórico-discursivos de cada género y las movidas características a la luz de los posibles lectores de cada uno de ellos. Pese a que la inclusión del género artículo de investigación enriquece la propuesta e intenta responder a las necesidades particulares de los estudiantes de doctorado, existen condiciones externas que obstaculizan el trabajo. En primer lugar, el curso tiene una extensión fija y común a todos los participantes, aunque estos se hallen en carreras o etapas distintas de sus estudios de posgrado, lo cual representa un impedimento para que algunos estudiantes puedan escribir sobre sus propios proyectos de investigación en inglés. Sumado a ello, existen diferencias notorias respecto del dominio de la lengua extranjera, así como de las instancias previas de enculturación disciplinar, aspectos que sin dudas afectan la escritura en inglés.

En línea con los aportes de Navarro, la enseñanza que se propone en 13 es articulada y socio-constructivista. A diferencia de las propuestas anteriores (17 e 12), 13 plantea desde el inicio del curso un análisis crítico-reflexivo sobre las posibles relaciones existentes 
Innocentini, V. (2021). La enseñanza de la escritura científico-académica en inglés en el posgrado: Reflexiones sobre la práctica. UCMaule, 67, julio-diciembre, 121-133. DOI: https://doi.org/10.29035/ucmaule.61.121

entre las elecciones lingüístico-discursivas de los escritores y las comunidades discursivas en las que se insertan. Se analiza el impacto de ciertas elecciones lingüísticas en los títulos, expresados como frases nominales o como oraciones, y se discuten además motivaciones posibles de ciertas elecciones discursivas de los escritores al publicar en inglés o al hacerlo en español, como estrategias docentes para articular los usos concretos de la lengua con sus contextos específicos de uso. La elección de ciertos recursos lingüísticos para realizar determinadas movidas retóricas en una u otra lengua es analizada en 13 con un objetivo descriptivo, explicativo, a diferencia de un enfoque más bien prescriptivo adoptado en la propuesta original (17). Por otra parte, aspectos centrales de la comunicación científica pasados por alto en 17 y en 12 , tales como el uso de recursos metadiscursivos (Hyland, 2019) para enfatizar o mitigar argumentos, son abordados explícitamente en 13 y representan estrategias para generar conciencia respecto del abanico de posibilidades que tienen a disposición los escritores y del alcance posible de tales elecciones. Dado que, como se mencionara anteriormente, muchos de los estudiantes de posgrado que toman los cursos de inglés carecen de un proceso adecuado de enculturación disciplinar y académica, el trabajo guiado con estrategias de refuerzo y mitigación en subgrupos promueve el aprendizaje colaborativo y el intercambio de opiniones de los participantes, quienes no sólo se limitan a analizar extractos de ejemplares escritos en ambas lenguas, sino que deben ajustar sus producciones escritas en función de situaciones hipotéticas planteadas. Pese a que tales situaciones no responden inicialmente a usos reales de los participantes, sino que son creadas con fines pedagógicos, los roles asumidos por los escritores representan un primer paso hacia la enculturación. Al compartir con sus pares y docente las elecciones lingüísticas y fundamentarlas, los estudiantes reflexionan acerca del lenguaje en uso. Un aspecto que deseo mencionar como limitación en 13 es la ausencia de un equipo docente interdisciplinario, que permita aportar una mirada más completa y más compleja de las posibilidades y restricciones lingüísticodiscursivas según las perspectivas de diversos actores de la comunidad discursiva (escritores experimentados, editores).

Finalmente, la propuesta de enseñanza plasmada en 13 y originada en iniciativas anteriores es explícita y promueve una mirada crítica. Desde la primera iniciativa (17) en adelante, los recursos y propuestas didácticas fueron diseñados a partir de un análisis exploratorio de un corpus de abstracts y artículos de investigación vinculados a los campos disciplinares de las carreras of recidas en la facultad. Sin embargo, mientras que el acercamiento a los géneros, y por tanto, el diseño de actividadesy demás, fue empírico e intuitivo en 17, 12 se diseñó partiendo de la literatura existente (Pho, 2008; Salager- 
Innocentini, V. (2021). La enseñanza de la escritura científico-académica en inglés en el posgrado: Reflexiones sobre la práctica. UCMaule, 67, julio-diciembre, 121-133. DOI: https://doi.org/10.29035/ucmaule.61.121

Meyer, 2008; Swales, 2004; Van Bonn \& Swales, 2007) y de un análisis sistematizado de las movidas retóricas y sub-funciones (Motta Roth \& Hendges, 1996, 2010; Swales \& Feak, 2009) de los abstracts. Asimismo, en el último curso, se retoma y enriquece el análisis de corpus, con aspectos que trascienden las elecciones lingüísticas y permiten vincular más aún a los textos con los escritores como hacedores de su propio discurso y como partícipes de una comunidad discursiva determinada. En este sentido, se busca promover una mirada crítica en los estudiantes, respecto de los textos analizados y de sus propias producciones. Se explicitan, como se mencionara anteriormente, patrones discursivos dominantes y realizaciones lingüísticas características en inglés a la vez que se analiza y discute la influencia de factores culturales, políticos, lingüísticos y disciplinares en las elecciones discursivas de los escritores. Se espera que el análisis crítico-reflexivo de los géneros expertos en los intercambios promovidos en clase logre generar conciencia en los estudiantes, escritores nóveles, no solo de las convenciones existentes, sino además de las posibilidades de elegir y de desarrollar sus propias voces como escritores, incluso al hacerlo en una lengua extranjera.

\section{CONCLUSIONES}

Se buscó en este trabajo reflexionar respecto de la aplicabilidad de un enfoque críticopragmático y basado en géneros discursivos para abordar la enseñanza de la escritura científico-académica en inglés en el nivel de posgrado. Se recurrió para ello a un análisis empíricoy situado de una serie de iniciativas docentes llevadas a cabo en una universidad argentina, que permitió identificar en la transición hacia la propuesta más reciente una aproximación a los géneros expertos en inglés que da cuenta de una mirada de la escritura como una práctica discursiva, situada y socialmente construida. Los cambios introducidos en el proceso de transición hacia la iniciativa más reciente permiten visibilizar convenciones genéricas y patrones retórico-discursivos predominantes pero también posibilidades de variación y, por tanto, de desarrollo de una voz propia y de participación genuina de cada escritor. Aunque las propuestas aquí abordadas son un puntapié inicial para acompañar a los estudiantes de posgrado en el proceso de escritura de géneros expertos en inglés, es necesario continuar y profundizar el camino iniciado. El trabajo conjunto e interdisciplinario con actores diversos de la comunidad científico-académica constituye sin dudas el primer desafío, pero representa también una oportunidad concreta para que los jóvenes investigadores se inserten y participen activa y efectivamente en una comunidad discursiva internacional. 
Innocentini, V. (2021). La enseñanza de la escritura científico-académica en inglés en el posgrado: Reflexiones sobre la práctica. UCMaule, 67, julio-diciembre, 121-133. DOI: https://doi.org/10.29035/ucmaule.61.121

\section{REFERENCIAS BIBLIOGRÁFICAS}

Bazerman, C., Little, J., Bethel, L., Chavkin, T., Fouquette, D., \& Garufis, J. (2005). Reference guide to writing across the curriculum. Parlor Press.

Bazerman, C., Little, J., Bethel, L., Chavkin, T., Fouquette, D. \& Garufis, J. (2016). Escribir a través del currículum. Una guía de referencia. Universidad Nacional de Córdoba.

Carlino, P. (2013). Alfabetización académica diez años después. Revista Mexicana de Investigación Educativa, 18(57), 355-381. https://www.redalyc.org/articulo. oa?id=14025774003

Carrasco Altamirano, A. C. \& Kent Serna, R. L. (2011). Leer y escribir en el doctorado o el reto de formarse como autor de ciencias. Revista mexicana de investigación educativa, 16(51), 1227-1251. https://www.redalyc.org/articulo. oa?id=14019203010

Cassany, D. \& Morales, O. (2009). Leer y escribir en la universidad: los géneros científicos. En D. Cassany (Comp.), Para ser letrados. Voces y miradas sobre la lectura, (pp. 109-128). Paidós.

Castelló, M. \& Donahue, C. (Eds.). (2012). University writing: Selves and texts in academic societies. Brill.

Colombo, L. \& Carlino, P. (2015). Grupos para el desarrollo de la lectura científico-académica: una revisión de trabajos anglosajones. Lenguaje, 43(1), 13-34. https://doi. org/10.25100/lenguaje.v43i1.4993

Corcoran, J. (2019). Addressing the "Bias Gap": A research-driven argument for critical support of plurilingual scientists' research writing. Written Communication, 36(4), 538-577. https://doi.org/10.1177/0741088319861648

Corcoran, J. \& Englander, K. (2016). A proposal for critical-pragmatic pedagogical approaches to English for research publication purposes. Publications, 4(1), 1-10. https://doi.org/10.3390/publications4010006

Creme, P. \& Lea, M. (2008). Writing at university: A guide for students. McGraw-Hill Education.

Englander, K. \& Corcoran, J. N. (2019). English for research publication purposes: Critical plurilingual pedagogies. Routledge. 
Innocentini, V. (2021). La enseñanza de la escritura científico-académica en inglés en el posgrado: Reflexiones sobre la práctica. UCMaule, 67, julio-diciembre, 121-133. DOI: https://doi.org/10.29035/ucmaule.61.121

Gómez Espinoza, L., Arreola Rico, R. L. y Hernández, C. (2020). Escritura académica. uCMaule, (59), 79-102. https://doi.org/10.29035/ucmaule.59.79

Hyland, K. (2019). Metadiscourse: Exploring interaction in writing (2nd ed.). Bloomsbury Publishing.

Hyland, K. (2004). Genre and second language writing. University of Michigan Press.

Lillis, T. (2001). Student Writing. Access, Regulation, Desire. Routledge.

Molina-Natera, V. (2012). Escritura a través del currículo en Colombia: situación actual y desafíos. Magis, Revista Internacional de Investigación en Educación, 5(10), 93-108. https://www.redalyc.org/articulo.oa?id=281024896006

Motta-Roth, D. (2008). Análise crítica de gêneros: contribuições para o ensino e a pesquisa de linguagem. DELTA: Documentação de Estudos em Lingüística Teórica e Aplicada, 24, 341-383. https://doi.org/10.1590/50102-44502008000200007

Motta-Roth, D. \& Hendges, G. (1996). Uma análise de gênero de resumos acadêmicos (abstracts) em economia, lingüística e química. Revista do Centro de Artes e Letras, 18(1-2), 53-90.

Navarro, F. (2019). Aportes para una didáctica de la escritura académica basada en géneros discursivos. DELTA: Documentação de Estudos em Lingüística Teórica e Aplicada, 35(2), 1-32. https://doi.org/10.1590/1678-460X2019350201

Parodi, G. (2010). Alfabetización académica y profesional en el Siglo XXI. Leer y escribir desde las disciplinas. Planeta.

Pho, P. D. (2008). Research article abstracts in applied linguistics and educational technology: A study of linguistic realizations of rhetorical structure and authorial stance. Discourse studies, 10(2), 231-250. https://doi. org/10.1177/1461445607087010

Russell, D. (2002). Writing in the academic disciplines. A curricular history (2nd ed.). Southern Illinois University Press.

Salager-Meyer, F. (2008). Scientific publishing in developing countries: Challenges for the future. Journal of English for Academic Purposes, 7(2), 121-132. https://doi. org/10.1016/j.jeap.2008.03.009 
Innocentini, V. (2021). La enseñanza de la escritura científico-académica en inglés en el posgrado: Reflexiones sobre la práctica. UCMaule, 67, julio-diciembre, 121-133. DOI: https://doi.org/10.29035/ucmaule.61.121

Swales, J. M., \& Feak, C. B (2009). Abstracts and the writing of abstracts. University of Michigan Press.

Swales, J. (2004). Research genres: Explorations and applications. Cambridge University Press.

Van Bonn, S. \& Swales, J. (2007). English and French journal abstracts in the language sciences: three exploratory studies. Journal of English for Academic Purposes, 6(2), 93-108. https://doi.org/10.1016/j.jeap.2007.04.001

Waigandt, D., Noceti, A. \& Lothringer, R. M. T. (2019). Writing for publication in English: Some institutional initiatives at the Universidad Nacional de Entre Ríos. En J. N. Corcoran, J., K. Englander \& L. M. Muresan (Eds), Pedagogies and Policies for Publishing Research in English (pp. 56-73). Routledge. 